\title{
Consideraciones actuales en la utilización de coronas unitarias libres de metal en el sector posterior
}

Current considerations in the use of free metal single crowns in the posterior area

Enrique Gabriel Castro-Aguilar ${ }^{1, a}$, Carlos Octavio Matta-Morales ${ }^{2, a, c}$, Oscar Orellana-Valdivieso ${ }^{23, b}$

\section{RESUMEN}

Actualmente se están desarrollando sistemas libres de metal para la confección de restauraciones protésicas que cumplan con altas expectativas funcionales, biocompatibilidad y estética. Sin embargo, a pesar de la introducción de nuevos materiales y avances tecnológicos en el proceso de elaboración, se ha cuestionado el comportamiento mecánico de las coronas unitarias en el sector posterior relacionado con fracturas de la cerámica feldespática de recubrimiento, para mejorar estas deficiencias, actualmente se están planteando soluciones como la confección de coronas de diseño anatómico y coronas monolíticas de adecuada translucidez con vitrocerámica de disilicato de litio y zirconia. El objetivo del presente artículo fue realizar una revisión exhaustiva sobre las consideraciones actuales en la utilización de coronas unitarias libres de metal en el sector posterior.

PALABRAS CLAVE: coronas dentales, cerámicas, estética dental. (DeCS, BIREME)

\section{SUMMARY}

Currently developing new metal-free systems for the fabrication of prosthetic restorations that meet high functional expectations, biocompatibility and aesthetics. However despite the innovation of new materials and technological advances in the development process, it has been questioned the mechanical behavior of single crowns in the posterior area related fractures of the veneering ceramic, to improve these defects, are currently proposing solutions such as making anatomical crowns and translucency monolithic crowns with ceramic lithium disilicate and zirconia This article aims to conduct a review of current considerations in the use of metal free single crowns in the posterior area.

KEYWORDS: Dental crowns, ceramics, dental aesthetics. (MeSH, NLM)

Cirujano Dentista, Egresado de la Facultad de Odontología, Universidad de la Amazonía Peruana. Iquitos, Perú.

Facultad de Estomatología Roberto Beltrán, Universidad Peruana Cayetano Heredia. Lima, Perú.

Especialista en Rehabilitación Oral.

Técnico dental del área de laboratorio CAD/CAM. 


\section{INTRODUCCIÓN}

El tratamiento protésico fijo más frecuente corresponde a la utilización de coronas unitarias en el sector posterior (1). Debido a la creciente demanda de los pacientes se han desarrollado materiales protésicos que cumplan con las expectativas funcionales, de biocompatibilidad y estéticas (2).

\section{REVISIÓN DE LITERATURA}

Las coronas de metal cerámica son consideradas en la actualidad el "gold estándar" por sus excelentes propiedades funcionales, predictibilidad a largo plazo y adecuada estética (3). Sin embargo, el margen de metal hace que sea difícil de imitar la apariencia de los dientes naturales especialmente en los biotipos periodontales delgados dando un aspecto gris azulado en los tejidos blandos circundantes (4). Otra deficiencia está relacionada con la translucidez en las situaciones de poco espacio, a pesar que este problema fue parcialmente resuelto hace 25 años con el desarrollo de un diseño de la estructura reducida y nuevas cerámicas de hombro (5). Así pues, las coronas libres de metal vienen logrando mejores resultados estéticos además de resolver otros problemas como galvanismo y toxicidad (6-8) (Figura 1).

Distintos tipos de cerámica se han introducido para la confección de coronas libres de metal. El primer intento fue el de fortalecer la cerámica de feldespato convencional mediante la adición de leucita (9). Los

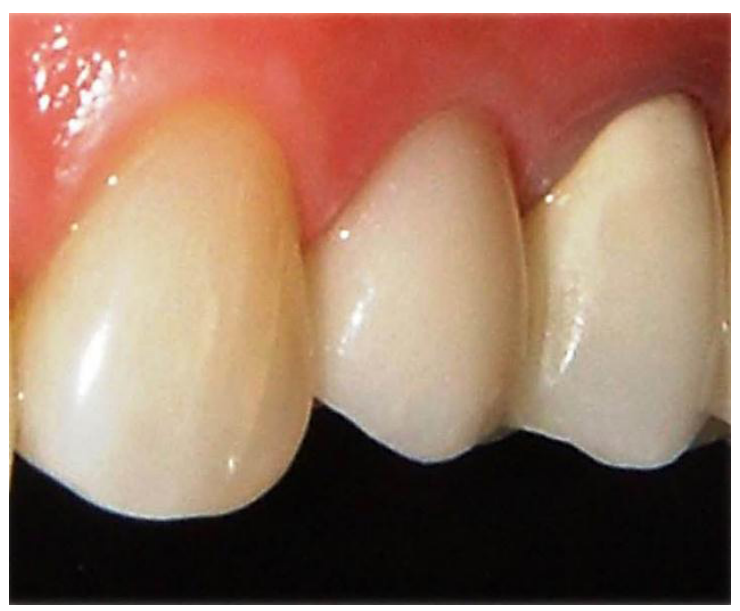

Figura 1. Nótese la opacidad en la corona metal cerámica y el halo gris en el margen gingival de la pieza 25 , mientras que la translucidez de la corona libre de metal de la pieza 24, le da una apariencia natural. resultados ópticos obtenidos con estos materiales eran similares a la apariencia dental natural. Sin embargo, debido a su baja resistencia mecánica y fragilidad estas cerámicas reforzadas fueron limitadas al uso exclusivo de coronas en el sector anterior (10).

Con el fin de combinar buenos resultados ópticos con mayor resistencia mecánica y estabilidad, se desarrollaron oxidocerámicas, dentro de las cuales destacaron la cerámica aluminosa de infiltración vítrea (In Ceram ${ }^{\circledR}$ Vita Zahnfabrik) y la alúmina densamente sinterizada (Procera ${ }^{\circledR}$ AllCeram, Nobel Biocare, Göteborg, Sweden), las cuales se caracterizaron por una mayor estabilidad estructural debido al alto contenido de matriz de alúmina cristalina mejorando las propiedades mecánicas; sin embargo, su alta opacidad limitó su uso solo para infraestructuras de coronas cubiertas con cerámica feldespática (11). A pesar del aumento de la estabilidad y resistencia del material, se registraron altas tasas de fracaso de las coronas unitarias en las regiones posteriores $(12,13)$.

Recientemente, dos materiales han demostrado éxito clínico a corto y mediano plazo para la confección de coronas unitarias libres de metal en el sector posterior; zirconia parcialmente estabilizada con itrio y la vitrocerámica de disilicato de litio (14). El objetivo del presente trabajo es hacer una exhaustiva revisión sobre las consideraciones actuales en la utilización de coronas unitarias libres de metal en el sector posterior.

\section{Zirconia}

El óxido de zirconio ó zirconia $\left(\mathrm{ZrO}_{2}\right)$ fue aislado por primera vez por el químico M.H. Klaproth en 1798 (15), este material se encuentra presente en la naturaleza en su fase monoclínica la cual necesita ser estabilizada en su fase tetragonal para ser usada en odontología, se encuentra disponible en el mercado como: zirconia parcialmente estabilizada con magnesio, zirconia reforzada con alúmina y zirconia parcialmente estabilizada con itrio, que es la más estudiada y difundida (16).

Para transformar la zirconia en fases de tipo tetragonal y cúbico es necesario aumentar la temperatura. El cambio de estructura es reversible y provoca cambios dimensionales que pueden producir grietas en el material. Cuando comienza a enfriarse, 
luego de haber atravesado altas temperaturas, se generan grandes tensiones que pueden llevar al óxido de zirconio puro a la fractura. El agregado de 2 a $3 \%$ de óxido de itrio estabiliza parcialmente la fase tetragonal y a partir del polvo base se confeccionan los bloques de zirconia estabilizada con itrio. El interés principal en el uso del óxido de itrio $\left(\mathrm{Y}_{2} \mathrm{O}_{3}\right)$ como estabilizante es la posibilidad de obtener cerámicas formadas casi completamente por zirconia

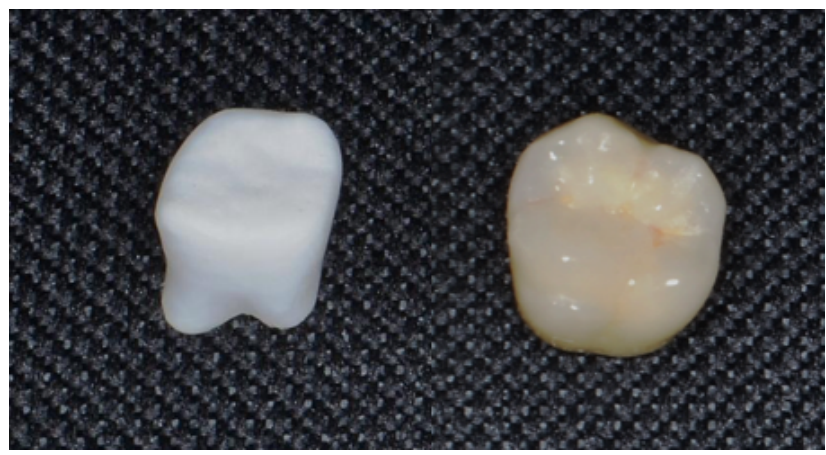

Figura 2. Infraestructura de zirconia y su posterior aplicación de cerámica feldespática de revestimiento.

en fase tetragonal a temperatura ambiente $(17,18)$. Las coronas de zirconia parcialmente estabilizada con itrio muestran propiedades mecánicas superiores, debido a valores de resistencia a la flexión y la resistencia a la fractura de $1600 \mathrm{MPa}$ y $9 \mathrm{MPa} / \mathrm{m}^{1 / 2}$ respectivamente (17) (Figura 2).

Existe escasa información con respecto a las coronas individuales de zirconia sin embargo algunos estudios muestran fallas relacionadas con fracturas de la cerámica feldespática de recubrimiento (19-21). Se han desarrollado métodos in vitro de pruebas de fatiga con cargas cíclicas donde se ha confirmado la susceptibilidad de las coronas de zirconia cubiertas con cerámica feldespática (22-23).

La fractura de la cerámica de recubrimiento en las infraestructuras de zirconia es un fenómeno complejo y existen teorías que explican esta deficiencia, tales como, las diferencias en el coeficiente de expansión térmica entre la infraestructura y la supraestructura, las tensiones térmicas de enfriamiento residual y el diseño de la infraestructura entre otros factores $(24,25)$. Se han planteado cambios específicos en el diseño de la infraestructura con el objetivo de optimizar el apoyo de la cerámica de recubrimiento así como la utilización de coronas monolíticas de zirconia translucida $(23,26)$.

\section{Zirconia de diseño anatómico}

El diseño inicial de las infraestructuras de zirconia se vio limitado por las características de diseño y software de los primeros programas de CAD (computed-aided design o diseño asistido por computadora). Esto permitió realizar únicamente modelados simplificados de la infraestructura, los cuales respetaban los parámetros mínimos de espesor para la resistencia de los mismos. Hoy en día se ha demostrado ese diseño como insuficiente y como un posible factor de altas tasas de fractura de las cerámicas de recubrimiento. Basándose en los hallazgos actuales, se requiere un diseño de la estructura anatómica aún más pronunciada llamada zirconia de diseño anatómico (27) (Figuras 3 y 4).

Los estudios de laboratorio (28) demuestran que un diseño de la estructura anatómica pronunciada y un período de enfriamiento prolongado conducen a una reducción significativa de la tasa de fractura de la cerámica de recubrimiento de coronas unitarias

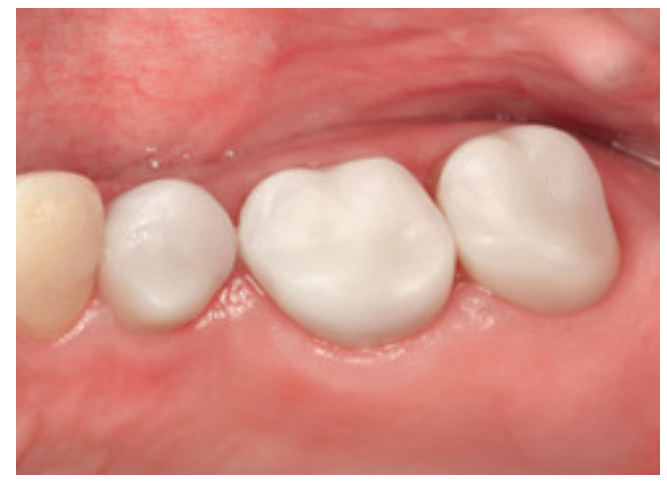

Figura 3. Infraestructura de zirconia de diseño anatómico. Nótese la importancia de mantener los contactos proximales a partir de esta fase.*

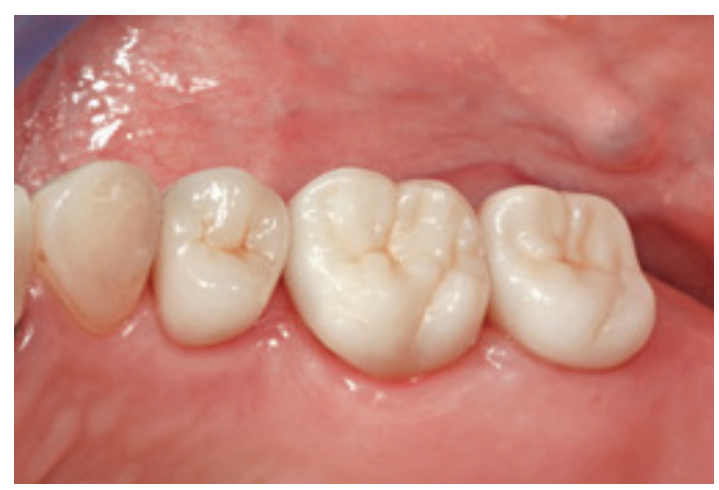

Figura 4. Coronas terminadas con la aplicación de carámica feldespática de revestimiento para conseguir una apariencia natural.* 
en el sector posterior (29). El diseño anatómico pronunciado requiere una adecuada reconstrucción de las infraestructuras en el ordenador CAD reduciendo significativamente el espesor de la cerámica de recubrimiento a nivel proximal proporcionando un máximo apoyo. Sin embargo, esto significa que las características estéticas de color no pueden ser alcanzados únicamente con las cerámicas de recubrimiento (30). En la actualidad se ha desarrollado la zirconia translucida a través de las siguientes tecnologías de producción: (31)

- Eliminación de la alúmina

- Aumento de las presiones de compactación uniaxial durante la fabricación

- Disminución de las velocidades de sinterización

- Aumento de las temperaturas de sinterización

La eliminación de la alúmina en la zirconia translucida disminuye en un $10 \%$ la resistencia mecánica pero aumenta la translucidez con respecto a la zirconia convencional permitiendo realizar coronas anatómicas o monolíticas con una resistencia a la flexión ligeramente inferior a la de zirconia convencional pero superior a una corona monolítica de vitrocerámica de disilicato de litio; sin embargo, la translucidez de este material no alcanza los valores de una vitrocerámica (30).

La elaboración de la forma anatómica final y caracterización del color individual se lleva a cabo con la aplicación de cerámica de recubrimiento o aplicación de tintes y la cementación se realiza de acuerdo al protocolo conocido para coronas de zirconia en la región posterior. (26)

\section{Zirconia de diseño de rebordes anatómicos "anatomic ridge design"}

Para las estructuras confeccionadas en zirconio ha sido propuesto un diseño, el cual ya ha sido utilizado en las restauraciones de metal cerámica, conocido como el diseño de rebordes anatómico "anatomic rigde design", cuyo objetivo consiste en mejorar la resistencia a la fractura de las cerámicas de revestimiento a través de los siguientes mecanismos (30):

- Aumentar la superficie de contacto entre la infraestructura y la cerámica de revestimiento
- Disipar las cargas oclusales, de manera que se transformen las cargas masticatorias horizontales y verticales (que se traducen en tracción y flexión) en fuerzas compresivas sobre las crestas de las infraestructuras.

Lamayor eficacia deldiseñoderebordes anatómicos con respecto al diseño anatómico convencional no ha sido demostrada; sin embargo, es una propuesta que pretende mejorar el comportamiento complejo de la unión infraestructura/cerámica de revestimiento propiciando una zona de soporte firme para evitar fracturas. (Figuras 5, 6 y 7).

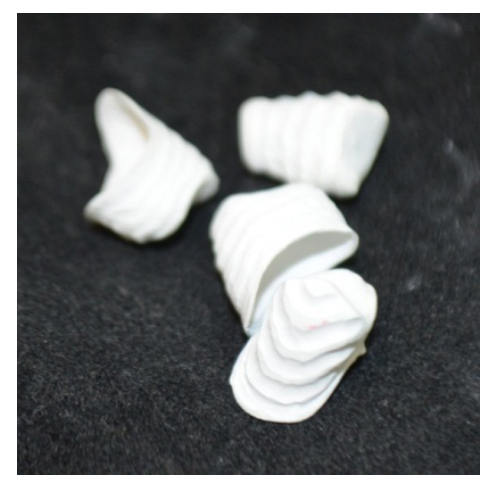

Figura 5. Infraestructuras pre-sinterizadas donde se diseñaron rebordes anatómicos ("anatomic rigde design") para mejorar el soporte de la cerámica de revestimiento.

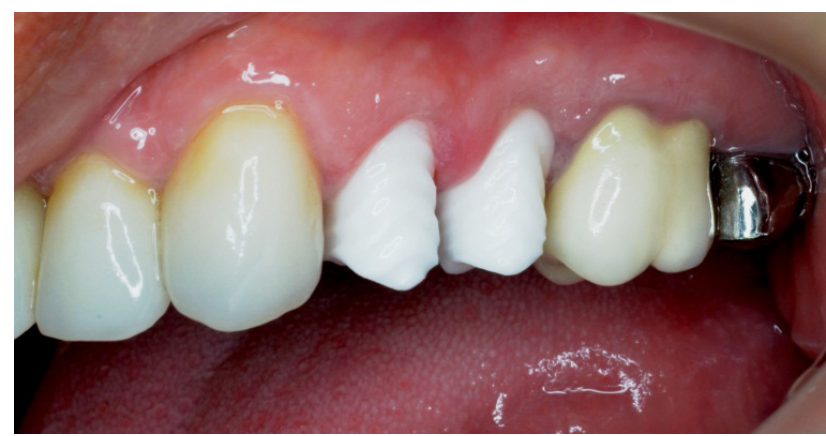

Figura6. Prueba de las infraestructuras sinterizadas.

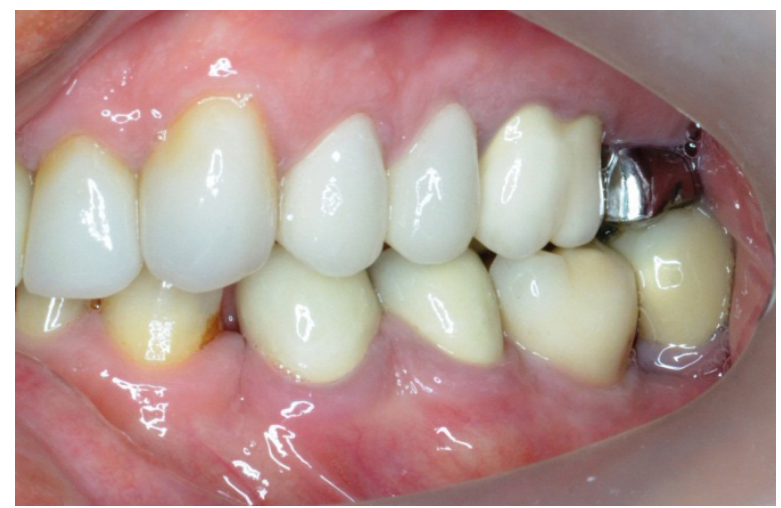

Figura 7. Coronas finalizadas. 


\section{Zirconia totalmente anatómica "monolítica"}

El uso de coronas de zirconia totalmente anatómicas en el sector posterior es una opción novedosa. La eliminación de la posibilidad de una fractura producida en la unión de la cerámica de recubrimiento es una clara ventaja. Por otro lado, la ventaja económica también es importante, ya que estas coronas se pueden producir con procedimientos de diseño y fabricación asistidos por computadora (CAD/CAM computed aided-design / computedaided manufacturing) a precios razonables. Por supuesto, la capacidad del software de diseño para generar una superficie oclusal completamente anatómica es una condición previa. Sin embargo, en lo que respecta a los aspectos clínicos, la posibilidad de generar coronas en espacio reducido y por lo tanto realizar preparaciones conservadoras en comparación con las restauraciones de coronas de zirconia con cerámica de recubrimiento, vienen a ser algunas de sus ventajas. De acuerdo con las instrucciones del fabricante, la reducción de la sustancia mínima puede ser de 0,5 a $0,7 \mathrm{~mm}$ en la zona oclusal y 0,5 $\mathrm{mm}$ en el contorno de la preparación. Sin embargo, estas recomendaciones se basan en los resultados de estudios in vitro del fabricante, que aún no han sido

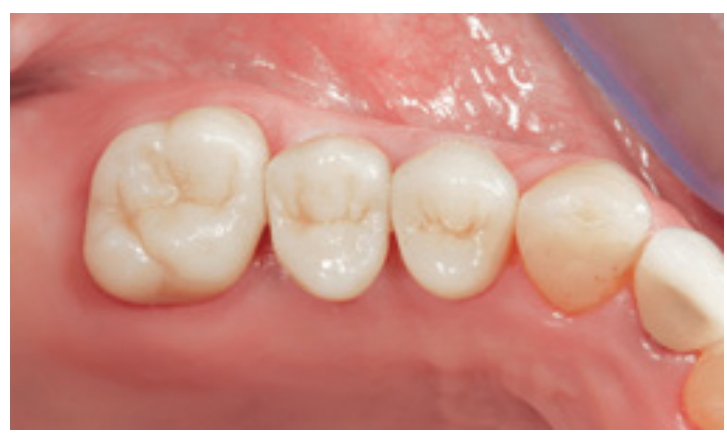

Figura 8. Coronas de zirconia totalmente anatómicas.*

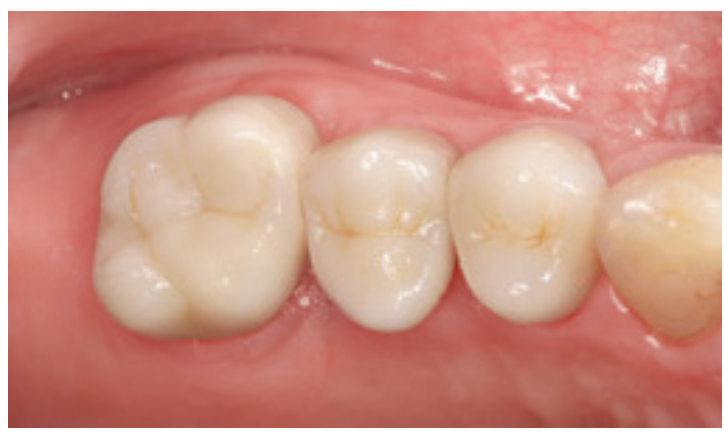

Figura 9. Coronas de zirconia totalmente anatómicas, después de haber sido maquilladas para lograr una apariencia natural.* verificados por estudios clínicos. Este proceso de confección de coronas monolíticas con reducciones de sustancia dental que antes solo podían lograrse con coronas de metal, debe realizarse con una selección cuidadosa del color de la estructura seguido del maquillaje individual (26) (Figuras 8 y 9).

En la actualidad, no existen datos de ensayos clínicos sobre coronas de zirconia totalmente anatómicas, sólo se han documentado reportes de casos con un período de observación de dos años $(32,33)$. Sin embargo, las ventajas clínicas y los riesgos potenciales se han evaluado exhaustivamente en estudios in vitro $(34,35)$.

El comportamiento de las coronas de zirconia totalmente anatómicas con respecto a la abrasión de las superficies del diente antagonista es esencial para la aplicación clínica, estos fueron estudiados por Preis y col. (36) y Rosentritt y col. (37) donde encontraron una menor capacidad abrasiva del esmalte del diente antagonista con las coronas de zirconia monolíticas en comparación a la vitrocerámica de disilicato de litio y la cerámica feldespática de recubrimiento debido a que estas últimas padecen una degeneración superficial que con el tiempo se transforma de una superficie lisa a una superficie con grietas y astillas perjudicando al esmalte.

El aumento de la acumulación de biopelícula es otro riesgo potencial en la aplicación clínica de las coronas de zirconia completamente anatómicas. Sin embargo, Bremer y col. (38) demostraron que las superficies de zirconia monolítica no muestran aumento de la formación de biopelículas en comparación con otros materiales cerámicos. Por lo tanto, teniendo en cuenta los aspectos científicos de los materiales, los estudios in vitro disponibles muestran aplicabilidad general de las restauraciones de zirconia totalmente anatómicas (39).

\section{Vitrocerámica de disilicato de litio}

La vitrocerámica de disilicato de litio es un material constituido por una cuota elevada de cristales agujiformes y entrelazados que obstaculizan la propagación de fracturas con resultados relativamente altos de resistencia a la flexión 350-400 $\mathrm{MPa} \mathrm{y}$ resistencia a la fractura $2-2.5 \mathrm{MPa} / \mathrm{m}^{1 / 2}(40)$.

La primera vitrocerámica de disilicato de litio (IPS 
Empress ${ }^{\circledR}$ II, Ivoclar Vivadent) fue introducida al mercado en 1998 con la limitación de que estaba indicada para la confección sólo de infraestructuras de coronas unitarias con la necesidad de una cerámica de recubrimiento (41). Los resultados de los estudios mostraron una tasa de fractura del $30 \%$ cuando se utiliza para la confección de coronas de dientes posteriores (42). La segunda generación de vitrocerámica de disilicato de litio (IPS e.max ${ }^{\circledR}$ Press, Ivoclar Vivadent) ofrecía cristales más pequeños y homogéneos con propiedades físicas mejoradas (resistencia a la flexión y resistencia a la fractura $10 \%$ superior al de su predecesor) (43). Dando la posibilidad de ser utilizada como infraestructura y coronas monolíticas procesadas a través de la técnica de inyección o por el diseño y fabricación asistido por computadora (CAD/CAM) con grados de translucidez alta (HT, high trasnlucency) y baja (LT, low translucency) y grados de opacidad media (MO, medium opacity) y alta (HO, high opacity) (44).

Las tecnologías para CAD/CAM utilizan una cristalización en dos fases que aprovecha las características del estado del disilicato de litio (45):

- Lafabricaciónindustrialdebloquesenbrutoaislados demetasilicatodelitioquegraciasalacristianización parcial son fácilmente fresables y permiten alclínico laspruebasdelascoronasconrespectoalaadaptación marginal, contactos proximales y oclusión directamente en la boca del paciente antes de la cristianización total.

- La cristianización final de las coronas fresadas mediante la cocción a $850{ }^{\circ} \mathrm{C}$ que en aproximadamente 35 minutos convierte al metasilicato de litio en disilicato de litio con una contracción mínima de $0.2 \%$ (Figuras 10, 11,12 y 13).

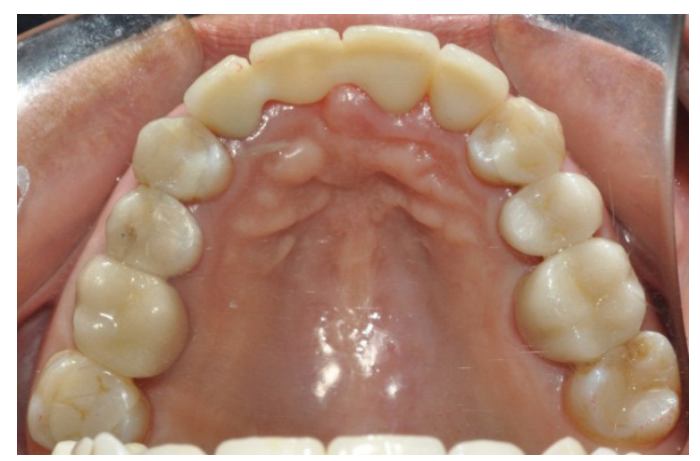

Figura 10. Prueba de las coronas de disilicato de litio monolíticas desarrolladas en CAD/CAM en su fase de cristianización parcial.

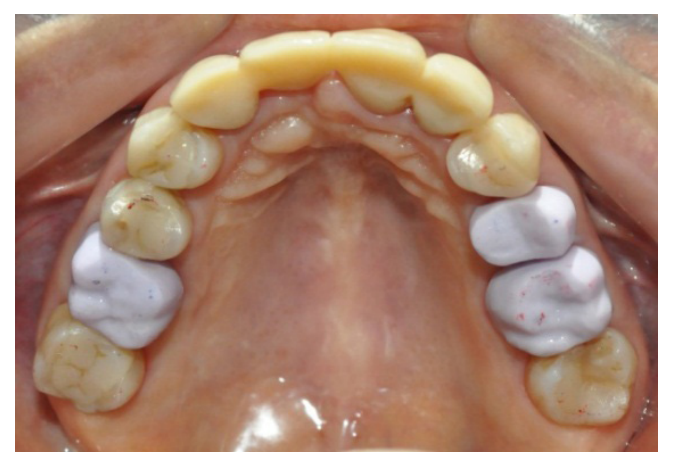

Figura 11. Coronas terminadas.

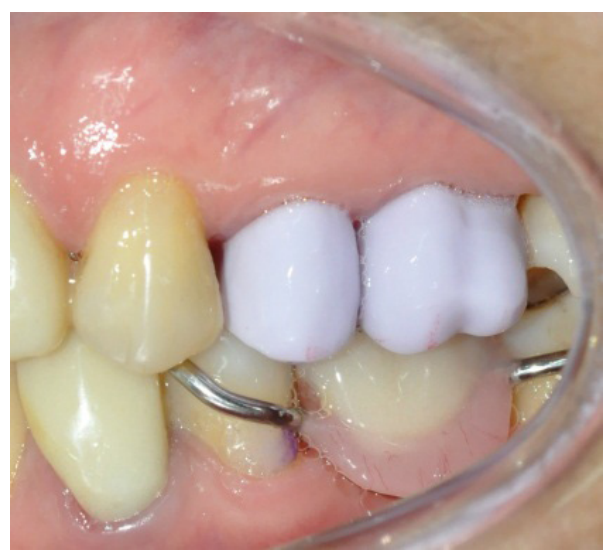

Figura 11. Vista lateral de las coronas terminadas.

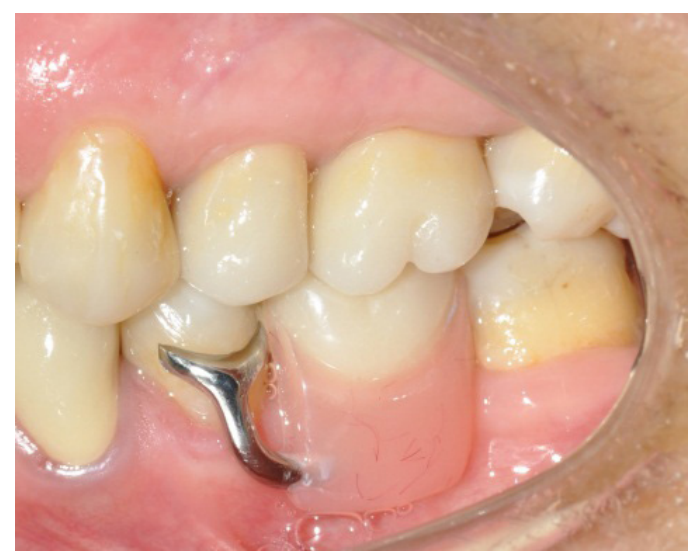

Figura 12. Vista lateral de las coronas de disilicato de litio nomolíticas en su fase de cristianización parcial.

Las coronas monolíticas de disilicato de litio han sido objeto de numerosos estudios comparativos in vitro contrastando los resultados de resistencia a la fractura superior a las coronas de zirconia convencional y similar a la coronas metal cerámica $(22,46,47)$. Debido a que su volumen se constituye en un $70 \%$ por cristales de matriz de vitrocerámica de granos finos, alargados de aproximadamente 1,5 
mm de longitud y $0,4 \mathrm{~mm}$ de diámetro, estos cristales alargados de disilicato de litio inhiben la propagación de fracturas las cuales se presentan solo en cargas muy altas en la fase de vidrio residual correspondiendo al $30-40 \%$ de su volumen. Esta microestructura permite explicar los valores elevados de resistencia a la fractura en una vitrocerámica (21).

Existen escasos ensayos clínicos prospectivos sobre la evaluación de coronas monolíticas de disilicato de litio, uno de ellos publicado por Reich y Schierz (48) donde se evidencia el éxito clínico del $96.3 \%$ después de 4 años de seguimiento. Otro estudio de Gehrt y Wolfart (49) evidencian el éxito clínico del $94.8 \%$ en coronas de disilicato de litio después de 9 años de seguimiento encontrando valores similares a las coronas de metal cerámica.

\section{CONCLUSIONES}

El proceso evolutivo de los sistemas libres de metal ha permitido el desarrollo de materiales que han demostrado éxito clínico para la confección de coronas unitarias libres de metal en el sector posterior, estos son; zirconia parcialmente estabilizada con itrio y la vitrocerámica de disilicato de litio.

A pesar de las ventajas mecánicas de la zirconia, se ha demostrado su susceptibilidad a las fracturas en la unión de la infraestructura con la cerámica de revestimiento relacionada con las diferencias en el coeficiente de expansión térmica entre la infraestructura y la supraestructura, las tensiones térmicas de enfriamiento residual y el diseño de la infraestructura.

Se han planteado cambios específicos en el diseño de la infraestructura de las coronas de zirconia con el objetivo de optimizar el apoyo de la cerámica de recubrimiento así como la utilización de coronas monolíticas de zirconia translucida obteniendo resultados promisorios en estudios in vitro.

Las coronas monolíticas de disilicato de litio utilizadas en el sector posterior ofrecen una opción de tratamiento con excelente resultado estético y de longevidad previsible con un alto pronóstico de éxito sustentado por ensayos clínicos prospectivos de 4 y 9 años de evaluación.

\section{Agradecimiento}

A la editorial Quintessence por ceder las fotos* del artículo: Rinke S, Fischer C. Range of indications for translucent zirconia modifications: clinical and technical aspects. Quintessence Int. September 2013; 44(8):557-66, para la ilustración del presente trabajo presentado en las figuras 3,4,8 y 9 cuyo contenido no presenta conflicto de intereses por parte de los autores y las cuales fueron cedidas gratuitamente por motivos estrictamente académicos.

Al Doctor Bulner Álvarez Paniagua y la Doctora Sally Ponce Apolinario por su aporte incondicional en la realización del presente artículo.

\section{Correspondencia}

Enrique Gabriel Castro Aguilar Calle Los Capulíes № 757, Departamento 302, Urb. La Aurora.

Miraflores-Lima 14, Perú.

Correo electrónico: castroaguilarenrique@gmail. com

\section{REFERENCIAS BIBLIOGRÁFICAS}

1. Dolan TA, Gilbert GH, Duncan RP. Foerster U. Risk indicators of edentulism, partial tooth loss and prosthetic status among black and white middle-aged and older adults. Community Dent Oral Epidemiol. 2001; 29(5):329-40.

2. Donovan TE. Factors essential for successful allceramic restorations. J Am Dent Assoc 2008; 139 Suppl:14S-18S.

3. Pjetursson BE, Sailer I, Zwahlen M, Hämmerle CH. A systematic review of the survival and complication rates of all-ceramic and metal-ceramic reconstructions after an observation period of at least 3 years. Part I: Single crowns. Clin Oral Implants Res. 2007; 18(3):7385.

4. Geller W. Dark and shaded zones-one of the important aspects of the W. Geller creative color technic. Quintessenz Zahntech 1982; 8:467-473.

5. Anusavice, K.J. Recent developments in restorative dental ceramics. J Am Dent Assoc. 1993; 124(2):72-4.

6. Raigrodski A, Chiche GJ. The safety and efficacy of anterior ceramic fixed partial dentures: a review of the literature. J Prosthet Dent. 2001; 86(5):520-5.

7. Dietschi D. Indications and potential of bonded metalceramic fixed partial dentures. Pract Periodontics Aesthet Dent. 2000; 12(1):51-8.

8. Näpänkangas R, Raustia A. An 18-year retrospective 
analysis of treatment outcomes with metal ceramic fixed partial dentures. Int J Prosthodont. 2011; 24(4):314-9.

9. McLean JW, Hughes TH. The reinforcement of dental porcelain with ceramic oxides. Brit Dent J. 1965; 119(6):251-67.

10. Dalloca L, Demolli U. A new esthetic material for anterior crowns: IPS-Empress. Quintessence of Dental Technology (QDT) 1995;18:171-175.

11. Magne P, Belser U. Esthetic improvements and in vitro testing of In-Ceram Alumina and Spinell ceramic. Int J Prosthodont. 1997; 10(5):459-66.

12. Sorensen JA, Choi C. Fanuscu MI, Mito WT. IPS Empress Crown system: three year clinical trial results. J Calif Dent Assoc. 1998; 26(2):130-6.

13. Sorensen JA, Kang SK, Torres TJ, Knode H. In-Ceram fixed partial dentures: three year clinical trial results. J Calif Dent Assoc. 1998; 26(3): 207-14.

14. Pjetursson B, Sailer I, Zwahlen M, Hämmerle C. A systematic review of the survival and complication rates of all-ceramic and metal-ceramic reconstructions after an observation period of at least 3 years. Part I: single crowns. Clin Oral Implan Res 2007; 18(3):7385.

15.Vilarrubi A. Pebé P, Rodriguez A. Prótesis fija convencional libre de metal: tecnología CAD CAM-Zirconia, descripción de un caso clínico. Odontoestomatología. 2011; 13(18):16-28.

16. Ali S, Karthigeyan S, Deivanai M, Mani R. Zirconia: properties and application -- a review. Pakistan Oral \& Dental Journal. 2014;34(1):178-183.

17. Vagkopoulou T, Koutayas S, Koidis P, Strub J. Zirconia in Dentistry: Discovering the nature of an upcoming bioceramic. Eur J Esthet Dent. 2009; 4(2):130-51.

18. Cehreli MC, Kokat AM, Akca K. CAD/CAM zirconia vs. slip-cast glass-infiltrated alumina/zirconia allceramic crowns: 2-year results of a randomized controlled clinical trial. J Appl Oral Sci. 2009; 17(1):4955.

19. Ortorp A, Kihl ML, Carlsson GE. A 3-year retrospective and clinical follow-up study of zirconia single crowns performed in a private practice. J Dent. 2009; 37(9):7316.

20. Groten M, Huttig F. The performance of zirconium dioxide crowns: a clinical follow-up. Int J Prosthodont. 2010; 23(5):429-31.

21. Coelho PG, Silva NR, Bonfante EA, Guess PC, Rekow ED, Thompson VP. Fatigue testing of two porcelainzirconia all-ceramic crown systems. Dent Mater. 2009; 25(9): 1122-7.

22. Coelho PG, Bonfante EA, Silva NR, Rekow ED, Thompson VP. Laboratory simulation of Y-TZP all-ceramic crown clinical failures. J Dent Res. 2009; 88(4):382-6.

23. Silva NR, Bonfante EA, Zavanelli RA, Thompson VP,
Ferencz JL, Coelho PG. Reliability of metalloceramic and zirconia-based ceramic crowns. J Dent Res. 2010; 89(10):1051-6.

24. Zarone F, Russo S, Sorrentino R. From porcelainfused-to-metal to zirconia: clinical and experimental considerations. Dent Mater. 2011; 27(1):83-96.

25. Alghazzawi T, Lemons J, Liu P, Essig M, Bartolucci A, Janowski G. Influence of low-temperature environmental exposure on the mechanical properties and structural stability of dental zirconia. Journal of Prosthodontics. 2012; 21(5):363-369.

26. Rinke S, Fischer C. Range of indications for translucent zirconia modifications: Clinical and technical aspects. Quintessence Int. 2013; 44(8):557-66.

27. Larsson C, Madhoun S, Wennerberg A, Vult von Steyern P. Fracture strength of yttria-stabilized tetragonal zirconia polycrystals crowns with different design: an in vitro study. Clinical Oral Implants Research. 2012; 23(7):820-826

28. Rues S, Kröger E, Müller D, Schmitter M. Effect of firing protocols on cohesive failure of all-ceramic crowns. J Dent. 2010; 38(12):987-94.

29. Rinke S, Schäfer S, Roediger M. Complication rate of molar crowns: a practice-based clinical evaluation. Int J Comput Dent. 2011; 14(3):203-18.

30. Montagna F, Barbesi M. Cerámicas, Zirconio y CAD/ CAM. 1ra. ed. Venezuela: Editorial Amolca; 2013.

31. Marchack BW, Futatsuki Y, Marchack CB, White SN. Customization of milled zirconia copings for allceramic crowns: a clinical report. J Prosthet Dent. 2008; 99(3):169-73.

32. Zhang Y. Making yttria-stabilized tetragonal zirconia translucent. Dental Materials. 2014; 30(10):1195-203.

33. Rojas-Vizcaya F. Full zirconia fixed detachable implantretained restorations manufactured from monolithic zirconia: clinical report after two years in service. J Prosthodont. 2011; 20(7):570-6.

34. Preis V, Behr M, Hahnel S, Handel G, Rosentritt M. In vitro failure and fracture resistance of veneered and full-contour zirconia restorations. Journal of Dentistry. 2012; 40(11):921-8.

35. Preis V, Weiser F, Handel G, Rosentritt M. Wear performance of monolithic dental ceramics with different surface treatments. Quintessence Int. 2013; 44(5):393-405.

36. Preis V, Behr M, Kolbeck C, Hahnel S, Handel G, Rosentritt M. Wear performance of substructure ceramics and veneering porcelains. Dent Mater. 2011; 27(8):796-804.

37. Rosentritt M, Preis V, Behr M, Hahnel S, Handel G, Kolbeck C. Two-body wear of dental porcelain and substructure oxide ceramics. Clin Oral Investig. 2012; 16(3):935-43.

38. Bremer F, Grade S, Kohorst P, Stiesch M. In vivo biofilm formation on different dental ceramics. Quintessence 
Int. 2011; 42(7):565-74.

39. Zhang Y, Lee J, Srikanth R, Lawn B. Edge chipping and flexural resistance of monolithic ceramics. Dental Materials. 2013; 29(12):1201-8

40. Reich S, Fischer S, Sobotta B, Klapper H, Gozdowski S. A preliminary study on the shortterm efficacy of chairside Computer-Aided Design/Computer-Assisted Manufacturing generated posterior lithium disilicate crowns. Int J Prosthodont. 2010; 23(3):214-6.

41. Wiedhahn K. From blue to white: new highstrength material for Cerec-IPS e.max CAD LT. Int J Comput Dent. 2007; 10(1):79-91.

42. Marquardt P, Strub JR. Survival rates of IPS empress II all ceramic crowns and fixed partial dentures: results of a 5-year prospective clinical study. Quintessence Int. 2006; 37(4):253-9.

43. Tysowsky GW. The science behind lithium disilicate: a metal-free alternative. Dent Today. 2009; 28(3):112-3.

44.Al Ben Ali A, Kang K, Finkelman M, Zandparsa R, Hirayama H. The effect of variations in translucency and background on color differences in $\mathrm{CAD} / \mathrm{CAM}$ lithium disilicate glass ceramics. Journal of Prosthodontics. 2014; 23(3):213-20.
45.Schultheis, S., Strub, J., Gerds, T., \& Guess, P. Monolithic and bi-layer CAD/CAM lithiumdisilicate versus metal-ceramic fixed dental prostheses: Comparison of fracture loads and failure modes after fatigue. Clinical Oral Investigations. 2013; 17(5):1407-13.

46. Guess PC, Zavanelli RA, Silva NR, Bonfante EA, Coelho PG, Thompson VP. Monolithic CAD/ CAM lithium disilicate versus veneered Y-TZP crowns: comparison of failure modes and reliability after fatigue. Int J Prosthodont. 2010; 23(5):43442.

47.Zhao K, Wei Y, Pan Y, Zhang X, Swain M, Guess $\mathrm{P}$. Influence of veneer and cyclic loading on failure behavior of lithium disilicate glass-ceramic molar crowns. Dent Mater. 2014; 30(2):164-71.

48. Reich S, Schierz O. Chair-side generated posterior lithium disilicate crowns after 4 years. Clin Oral Investig. 2013; 17(7):1765-72.

49.Gehrt M, Wolfart S, Rafai N, Reich S, Edelhoff D. Clinical results of lithium-disilicate crowns after up to 9 years of service. Clin Oral Investig. 2013; 17(1):275-84.

Recibido: 14/01/2014

Aceptado: 30/03/2014 